\title{
The assembly dimension control technology of stainless steel pipe and copper fins for thermal shroud
}

\author{
Fei $\mathrm{Xu}^{1, \mathrm{a}}$, Yuzhong $\mathrm{Li}^{2, \mathrm{~b}}$, Wanqing Yang ${ }^{3, \mathrm{c}}$, Yongli Tong ${ }^{4, \mathrm{~d}}$, Lijun Cui ${ }^{5, \mathrm{e}}$ \\ ${ }^{1}$ Beijing Institute of Spacecraft Environment Engineering, China \\ ${ }^{2}$ Beijing Institute of Spacecraft Environment Engineering, China \\ ${ }^{3}$ Beijing Institute of Spacecraft Environment Engineering, China \\ ${ }^{4}$ Beijing Institute of Spacecraft Environment Engineering, China \\ ${ }^{5}$ Beijing Institute of Spacecraft Environment Engineering, China \\ abuaaxf@163.com, blyzcasc@126.com, yangwq@163.com, ${ }^{\mathrm{d}} \mathrm{TYL} @ 126 . c o m,{ }^{\mathrm{e}}$ cuilijun@126.com
}

Keywords: stainless steel pipe and copper fins; thermal shroud; assembly technology.

\begin{abstract}
The thermal shroud has the structure of stainless steel pipe welding with copper fins. It has well capability of heat exchange, structural strength and inflexibility which is used to many types of equipment for space environment simulation in recent years. This article will conduct technology research for the key processing sectors of such large-scale thermal shroud and dimension control technology. Based on the analysis of the structure and layout of the thermal shroud, discussed the lifting, installation and the fixture. Finally the reliability and validity were verified by the actual processing of the thermal shroud and the future improvement and development of this type of the thermal shroud were made.
\end{abstract}

\section{Foreword}

The main role of the thermal shroud is to produce cold and dark space environment in the space environment simulation equipment ${ }^{[1,2]}$. Because of the thermal shroud works in a vacuum environment and alternating high and low temperature conditions, so the thermal shroud material and structure must be able to withstand high and low temperatures $\left(-196^{\circ} \mathrm{C} \sim+100^{\circ} \mathrm{C}\right)$ and other conditions requirements like alternating temperature ${ }^{[3,4]}$. At the same time the requirement for the cold and black environment provided by the thermal shroud must be temperature distribution, so the overall heat transfer performance of the thermal shroud have to be good $^{[5]}$. In a large space environment simulator, the thermal shroud, which is generally formed by the tube and splice plate structure, simulates cold and black space environment. Its inner surface is coated with black paint which has high absorption rate, making the absorption rate to 0.9 or more, and its tube is passed into liquid nitrogen, so that the whole thermal shroud is maintained at a temperature below $100 \mathrm{k}^{[6,7,8]}$. The thermal shroud has the structure of stainless steel pipe welding with copper fins. It has an strong overall rigidity and good cooling effect which is particularly suitable for the large thermal shroud with more than three meters in diameter. It has been successfully applied to China Academy of Space Technology KM3B ${ }^{[9]}$, Shanghai Academy of Spaceflight Technology KM2\&KM5, KM3C, KM3D, KM3F and other large environmental simulation equipment ${ }^{[10]}$.

The key aspects of the thermal shroud manufacturing process of this structure include heterogeneous metal welding of stainless steel and copper fins, large-scale assembly tooling, cold shock, annealing, helium mass spectrometer leak detection, X-ray detection, black lacquer spraying. This article focuses on one - large tooling assembly to start on. The article first analyzes the structural characteristics of the thermal shroud, and then processed in accordance with the skeleton, the skeleton assembly and the manifold assembly. This three part elaborates thermal shroud assembly manufacturing faced challenges and solutions to ensure that the size, and introduces an auxiliary tooling and the use of a spreader. 


\section{Structure and layout of thermal shroud}

The thermal shroud of stainless steel and copper fins generally cylindrical horizontal structure, divided into door, cylinder and rear three parts. Each part has a relatively independent circulation line. The cylinder thermal shroud will be discussed mainly in this article.

Currently, the structure of thermal shroud is used for fish bone shape. There arranged two $\mathrm{LN}_{2}$ collecting pipes up and down. The two pipes are arranged in parallel between a numbers of finned pipes. The inlet collecting pipe is located bellow and another is located above, as shown in Fig. 1. The thermal shroud located skeleton, which mostly is annular structure. It is PTFE plate that isolated to adiabatic between the thermal shroud and the skeleton.

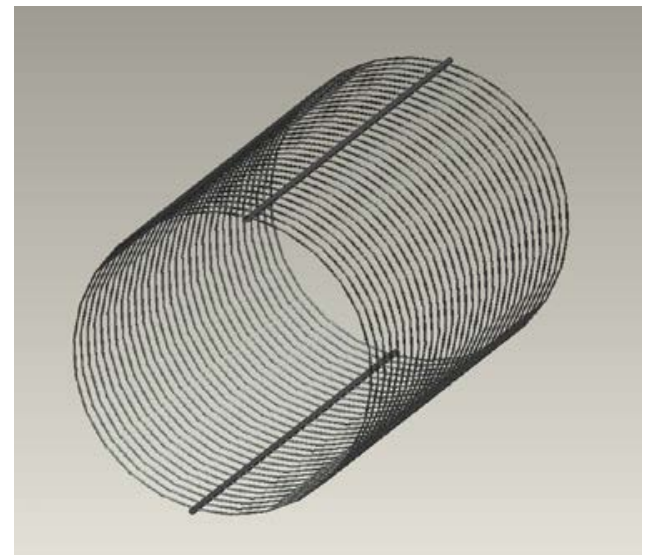

Fig. 1 layout of thermal shroud

Fins are welded from stainless steel pipe and copper plate. It is used in TIG welding without filling wire, which is used melted copper to bond steel surface, so that the tube is not penetration and leak. The copper fins must be annealed and liquid nitrogen cold impact test after welding is completed. It is a rounded shape with rounded machine after passing through the helium leak. At both ends of the tube head is vertical segment, which is for inserting summary tube.

\section{Dimension control technology in processing and assembly of thermal shroud}

\section{Dimension control for welding skeleton}

The thermal shroud frame is produced by with 0Cr18Ni9 plate, welded into a T-ring structure. The difficulty lies in stud processing of ring beam skeleton.

The first execution is to round plate. Rounding need two steps. Firstly, get out of the approximate shape of an arc on Rounder, then draw a circle on the table to be amended. You need be inside of the welding arc at the plate in a perfect circle in advance when corrected, and then round off work piece fixture plate fixed on the arc, and finally process into semi-circular arc stud against the inside of the plate. It is time to weld. At this point, you need a little finishing pad to be an auxiliary fixture. Then place studs on a small pad. This simple way both to ensure accurate positioning studs, but also ensures the verticality. It is as shown in Fig. 2.

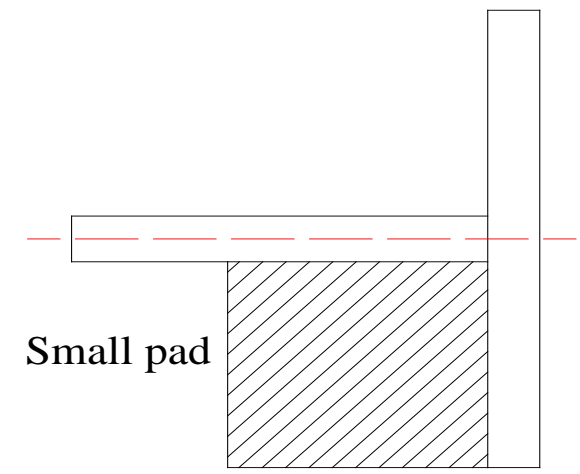

Fig. 2 Use Small pad in studs processing

In order to reduce welding distortion, ensure verticality studs, we use the form of phase soldering. Weld $100 \mathrm{~mm}$ per $100 \mathrm{~mm}$ interval, and then weld $100 \mathrm{~mm}$. Flip the skeleton after the 
completion of the welding side. In order to ensure the welding at the intersection of state for counteracting the welding deformation on both sides, we need weld on the other side after fixing clamp, as shown in Fig. 3.

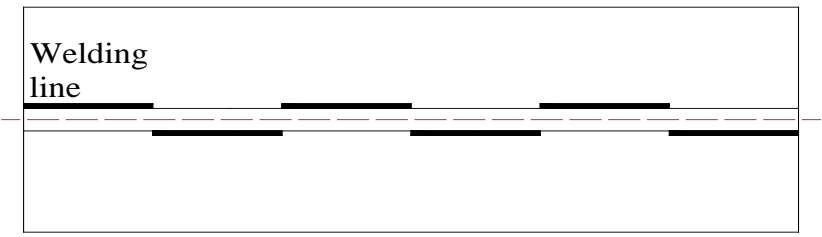

Fig. 3 Cross-welding of skeleton

\section{Dimension control for Cylinder skeleton assembly}

It is the whole assembly stage after finned tubes and the main branch pipe of the thermal shroud processing is complete. The assembly of cylinder skeleton is the key. Generally, the ring beam will be placed 4 or 5 . The ring beam is fixed by welding connecting plates. Due to the larger size of the cylinder, coupling with the presence of welding deformation, the overall structure size, horizontal, vertical are difficult to control, we need strict process to ensure the size.

First place pipe size $\Phi 80 \mathrm{~mm} \times 6 \mathrm{~mm}$ in the ring beam center position during cylinder skeleton assembly. And then with a square tube cross welding, spot welding can be easy removal, which is used for the whole skeleton in later adjusted, as shown in Fig. 4.

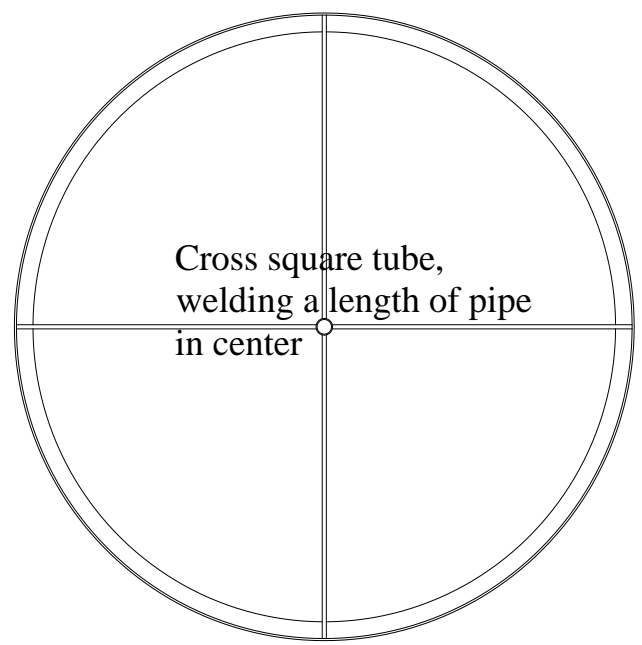

Fig. 4 Circular ring beam

The four ring beams are lifted, then each ring beams are in a free state. To make the next step to connect the beam welding, we must ensure two points: degree vertical beam and floor and each ring beam having to be at the same height. To do this, place a wide of $180 \mathrm{~mm}$ u-steel to bottom down, and adjust so that each ring beam can contact both sides of the u-steel. Then use spot welding to fix.

When connected to the T-shaped beam and ring beam by welding, we adopt the way of spot welding first, then adjustment, and full welding last. First in the middle of the ring beam insert a $\Phi$ $60 \mathrm{~mm} \times 5 \mathrm{~mm}$ tube, and then rotate each ring beam to circumferentially aligned. Casters installed at both sides of the skeleton are connected to one side of an aluminum tube. Holes of casters and holes of aluminum square tube are with punch. T-shaped connecting beam welding starts with the bottom of the barrel. Use the beam of spot welding to ensure the total size and the size of each segment of each stage to meet the requirements. Then flip the whole $180^{\circ}$ by the middle of the tube, followed by welding.

Make the first size adjustments at this time. Diagonal measurement of T-shaped connecting beam see whether they have the same length or not, as shown in Figure 5. If there is a deviation, then through a regulator adjust to ensure the overall in order. 


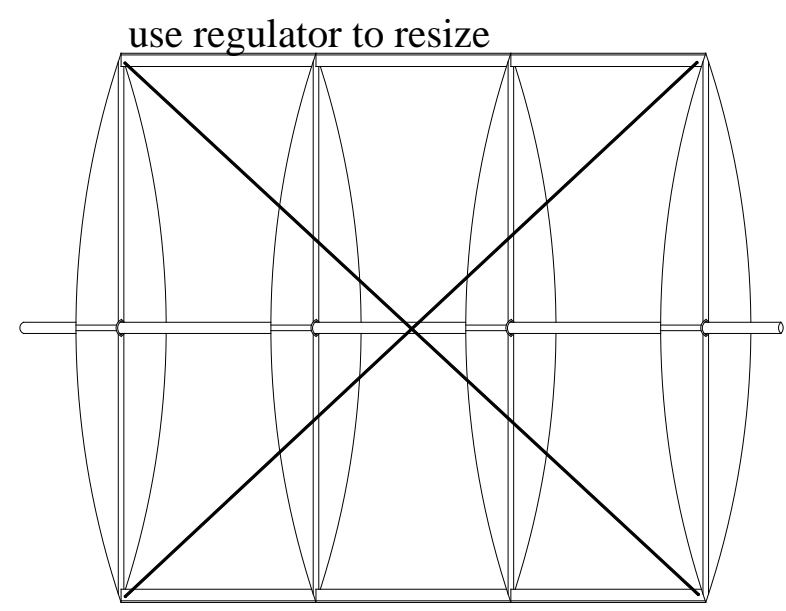

Fig. 5 First resizing (thick lines are regulator)

Then remove the two aluminums tube at castors, and welding the T-shaped connecting beam in this location, a total of 4 connecting beam has been welded at this time, the overall framework has become, take the integral framework transport to specially designed rotation fixture, to make the cylinder rotate and eliminate danger of welding operation at high place.

The rotation fixture as shown in Fig. 6

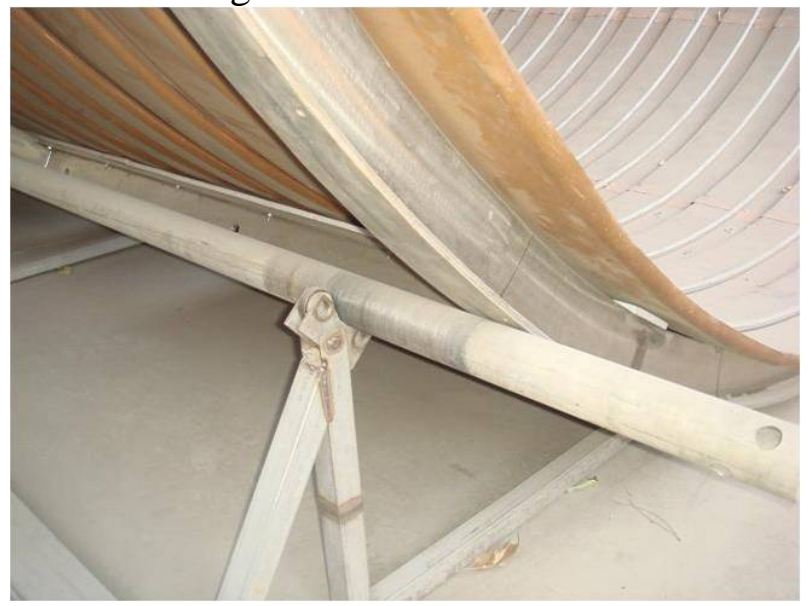

Fig. 6 Rotation fixture (part)

After welding the rest of T-shaped connecting beam, with the first adjustment similar. Both groups of the connecting beam frame were measured to test whether the square was founder, which is as long diagonal. If there was unequal measure, the second adjustment needs to do by a regulator until each group had as long diagonal. At this point dismantling the pipe and square tube frame of the central beam, full welding need to do at all spot welding, the whole skeleton was welded completely.

\section{Dimension control for branch pipe assembly}

The manifolds assembly process occurs after that the collecting pipe and the skeleton were completed, which was stainless tube and copper fins welded structures, as shown in Fig. 7. There are about 60 branch pipes in cylinder fins up and down, inserted in the collecting pipe, and then fixed by welding. To ensure that all branch pipes were arranged in uniform, neat, on one hand, all pipes cutting size should be the same. Copper fins were welded according to uniform standards, so that all the manifolds remain the same size. Another side was the tube head scribe; the insertion depth in the collecting pipe was controlled to be the same, taking into account the aggregated pipe thickness $2.5 \mathrm{~mm}$, general distance pipe end $3 \mathrm{~mm}$.

When installing the manifold, the first tube position is critical, which is the installed base of all other manifolds. When stalling the branch pipe was installed from the bottom, a rectangular device needs to be used to ensure the vertical between the branch pipe and the summary pipe. While a 
downward force is applied to the manifold to ensure the circular surface of manifold close to the ring beam skeleton. The order of the branch pipes was that six branch pipes installed firstly, and then measured the overall offset. If there was any offset, it needs to be corrected in time. The cylinder rotated $180^{\circ}$ and install another 12 pipes, and then measured, rotated $180^{\circ}$ and install 12 tubes, and so on. The purpose was eliminating potential safety hazards, because the cylinder was difficult to control for top-heavy when flipping up after loading a single side of the manifold.

The above process, the processing process of the control insert summary tube is a difficult, because all the manifolds rely on manual installation. Due to the limitation of ring beam skeleton and the depth was difficult to control after inserting a summary tube, there are some difficulties when the shaft hole match. In the technology, advanced process of enter firstly and then out was used. Firstly one end of the manifold inserted the summary deeper, so that another section located hole out. At the same time another section was fixed on the outside of the manifold with special fixture, it was inserted to dash of the hole along the arc slowly and then weld to fix, as shown in Fig. 8.

After all the branch pipes are installed, take the branch pipe and collecting pipe of the fillet weld full welding. When welding, in order to ensure the qualified $\mathrm{X}$ ray flaw detection and air tightness requirements, we need to take the following welding process:

1) In summary pipeline internal through argon protection, to prevent producing impurities by the reaction of welding and gas in air, affecting the quality of welding.

2) Polished pipe groove in advance, to guarantee the welding wire filling.

3) Cleaning before welding, using alcohol or acetone to remove (clear) oil and impurities.

4) Adjusting the process parameters, to achieve the effect of one side welding, double-sided molding.

5) Need full welding two, namely two layers, in order to ensure the strength of welding and sealing tightness.

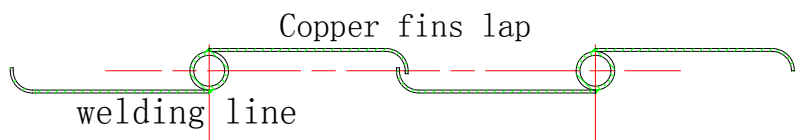

Fig. 7 Structure and layout of stainless steel and copper fins

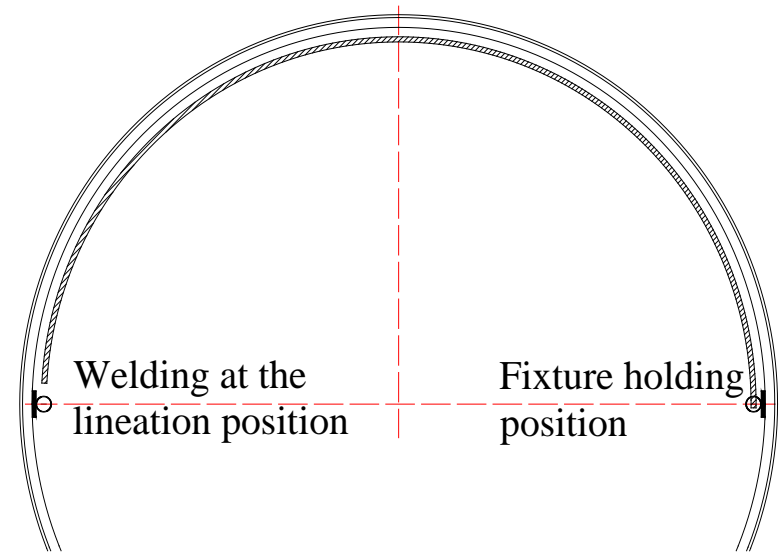

Fig. 8 Sketch map for branch pipe assembly

\section{The actual processing of thermal shroud}

This article takes a heat sink which is manufactured by a domestic institution for instance to illustrate the situation of the showed three terms of automatic welding which is used in this kind of thermal shroud.

\section{Dimension control for welding skeleton}

We use manual TIG welding to process the skeleton of this thermal shroud. Welding on both sides at staggered times makes the measured diameter of the skeleton of the ring beam to 3,099 which has a design size of 3,100 with an error less than $5 \mathrm{~mm}$. Vertical angle of vertical ribs is $88^{\circ}$ 
with an error less than $2^{\circ}$. As shown in Fig. 9.

\section{Dimension control for Cylinder skeleton assembly}

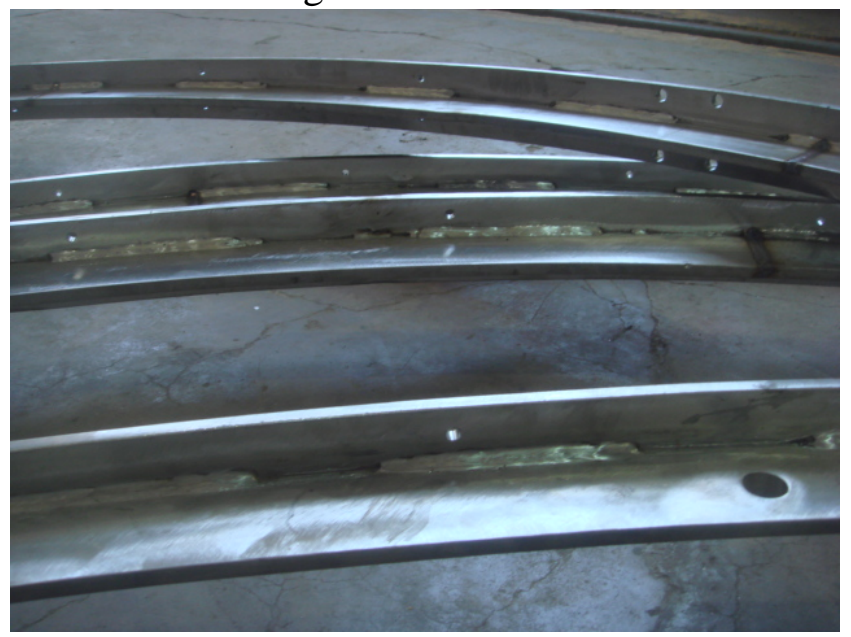

Fig. 9 The ring beam skeleton after processing

Assembly of cylinder skeleton is supported by ring beam cross, central pierced tube, using spot welding, measurement, adjustment and full-length welding. The total length of the ring beam skeleton after processing is $5,685 \mathrm{~mm}$, plan size is $5,690 \mathrm{~mm}$ with an error less than $10 \mathrm{~mm}$, the stayed length of regulator is $6,565 \pm 5 \mathrm{~mm}$. Straightening of the thermal shroud is showed in Fig. 10 .

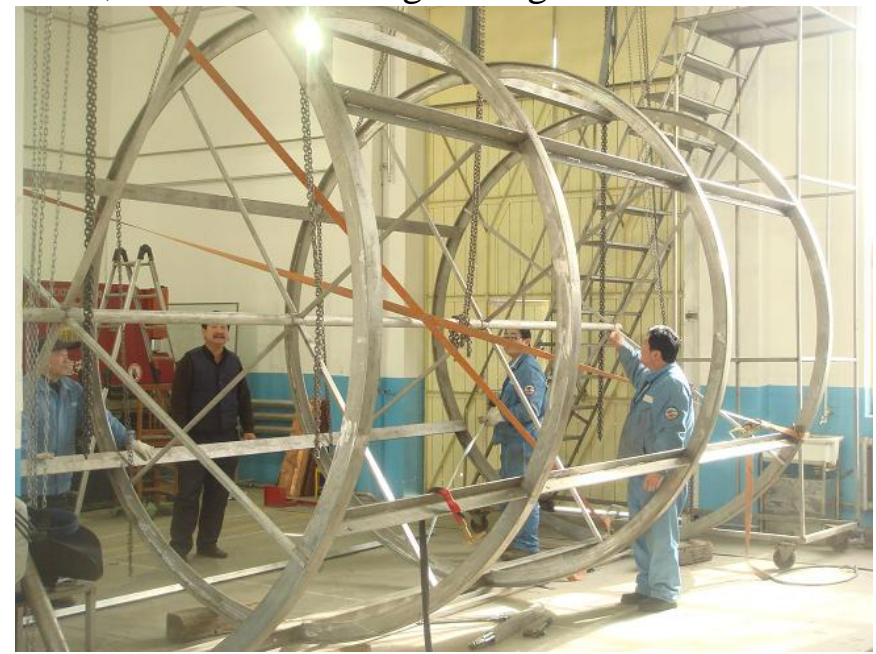

\section{Dimension control for branch pipe assembly}

The branch pipe assembly in accordance with the order of 6,12,12,12 under the process of equal size cutting and tube head scribing, make each branch pipe laps successively tight and arranges neatly. The edge height of pipe fins and the curvature of branch pipe consistent and the branch pipe is a tight fit to the skeleton of ring beam. As shown in Fig. 11 and Fig. 12.

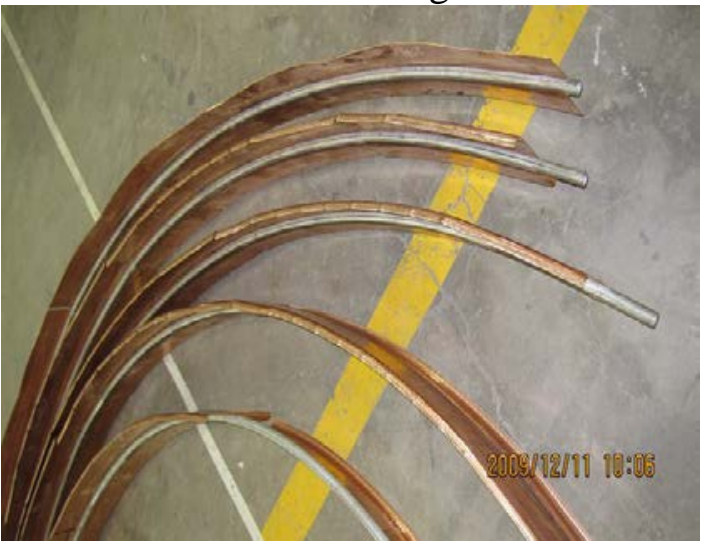

Fig. 11 The branch pipe fins

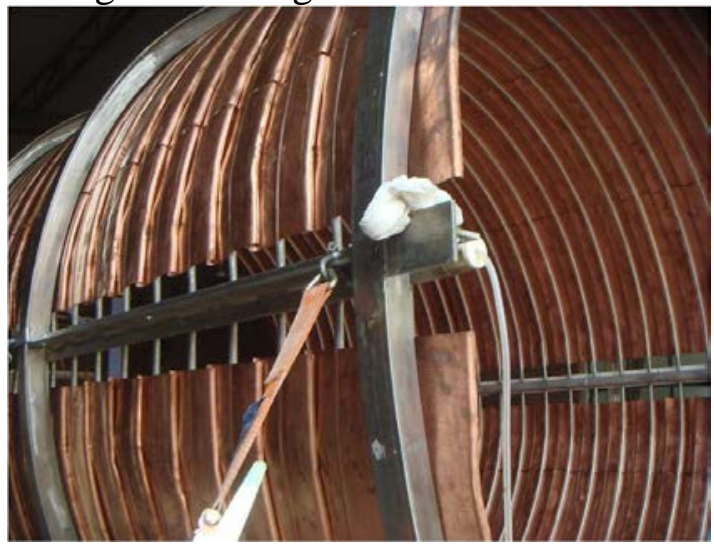

Fig. 12 The thermal shroud cylinder 


\section{Brief summary}

By studying the implementation of the above technology and methods, the thermal shroud has been completed pass helium mass spectrometer leak detection, pipes are all qualified, all welding line have passed the X-ray inspection and various sizes of the thermal shroud's shape are consistent with drawing. All of them meet the design requirements. Therefore, it is reliable to apply the dimension control technology to the stainless steel pipe and copper fins thermal shroud.

\section{Conclusion}

Basic on the automatic welding, this article discusses several key processing technologies, focuses on difficulties and solutions in the process of machining and assembling skeleton and fin branch pipes. This kind of stainless steel pipe and copper fins which has broad application prospects and lots of advantages including a large used space, well structure strength and inflexibility, higher cooling speed is a commonly used low-temperature vacuum test equipment in recent years. It can be proved in some aspects, such as the overall shape of skeleton, the structure of copper fins and automatic welding, for the purpose of reducing manufacturing cycle, solidify welding parameters to improve automation of machining thermal shroud and reduce relying on workers.

\section{References}

[1] Bencheng Huang: Space Environment Simulator Design. Beijing: Astronautic Publishing House, (1990) (In Chinese)

[2] Renhai Wei, Lijian Zhang, Xu Liu: Automatic welding with stainless steel pipe and copper fins for thermal shroud. Spacecraft Environmental Engineering, Vol. 27 (2010), p. 496-499 (In Chinese)

[3] Liwei Zhang, Wenjie Zhang, Renhai Wei, etc: The manufacturing technique of stainless steel pipe and copperplate structure in heat sink. Spacecraft Environmental Engineering, Vol. 25 (2008), p. 587-590 (In Chinese)

[4] Li Wang, Weijun Chen, Baoxiang Jiao, etc: Design of stainless steel-copper heat sink in manned module. Cyrogenics, Vol. 4 (2003) (In Chinese)

[5] Tao Yu, Min Liu, Dingzhong Zou: Thermal Uniformity Analysis for Heat Sink of Space Simulator. Chinese Space Science And Technology, Vol. 26 (2006), p. 37-41 (In Chinese)

[6] Hangsheng Zhou, Huisheng Zhang, Hengguo Mao: Design and research of heat accumulation device in large space environment. Cryo \& Supercond, Vol. 37 (2010), p. 11-15（In Chinese）

[7] Dingzhong Zou, Min Liu, Guoqing Liu: Design of a Thermal Shroud Attached to KM6 Space Simulator. Chinese Space Science And Technology, Vol. 22 (2002), p. 20-26 (In Chinese)

[8] Min Liu, Dingzhong Zou, Guoqing Liu: Welding Technique of Aluminum Heat Sink. Missiles And Space Vehicles, Vol. 1 (2001), p. 43-46 (In Chinese)

[9] Botao Liu, Xiaoqin Ru, Liwei Zhang: Development of KM3B Space Simulator. Spacecraft Environmental Engineering, Vol. 23 (2006), p. 232-235（In Chinese )

[10] Xiaoqin Ru, Botao Liu, Jinsong Liu: Development of space environmental simulator GVU-600. Spacecraft Environmental Engineering, Vol. 29 (2012), p. 667-670（In Chinese） 